\title{
DIE ERSTELLUNG DES LERNMEDIUMS ZUM THEMA "ERFINDUNGEN UND ERFINDER" AUF DEM BUCH STUDIO D A2 MIT WINDOWS MOVIE MAKER
}

\author{
Danita Silitonga \\ Rina Evianty \\ Tanti Kurnia Sari
}

\begin{abstract}
AUSZUG
Das Ziel dieser Untersuchung ist es, um das Lernmedium zum Thema „Erfindungen und Erfinder" aus dem Buch Studio d A2 mit Windows Movie Maker zu erstellen. In dieser Untersuchung wird die deskriptive qualitative Methode angewendet. Der Prozess der Erstellung des Lernmediums zum Thema ,Erfindungen und Erfinder"aus dem Buch Studio d A2 mit Windows Movie Maker besteht aus der Erklärung der Phasen von Richey und Klein. Darunter sind: (1) die Planung, (2) die Herstellung, (3) Evaluation/Bewertung und Revision. Die Daten in dieser Untersuchung sind Bilder und Wörter aus dem Buch Studio d A2. Diese Untersuchung wird in der Bibliothek an der Fakultät für Sprachen und Kunst an der Staatlichen Universität von Medan durchgeführt. Das Ergebnis der Erstellung des Lernmediums zum Thema „Erfindungen und Erfinder”aus dem Buch Studio d A2 mit Windows Movie Maker sind das Thema „Erfindungen und Erfinder” (Das Aspirin, Das Automobil, Der Buchdruck, Der Klettverschluss, Der Dieselmotor, Der Dübel, Der Fernsehen, Der Hubschrauber, Der Kaffefilter, Das Motorrad, Das MP3-Format, Die Schriffsschraube, Die Strassenbahn, Der Teebeutel, Die Zahnpasta), die Grammatik (Nebensätze um zu/damit, Konjugation Verb "werden", Passiv mit werden/wurden) und Übungen. Das Lernmedium mit Windows Movie Maker hat von einem Prüfer korrigiert, deshalb hat dieses erstellte Lernmedium Windows Movie Maker sehr gut. Hoffentlich kann diese Abschlussarbeit nützlich sein, um unser Wissen zur Wissenschaft über Lernsoftware, vor allem Deutschlernenden anzureichern.
\end{abstract}

Schlüsselwörter: Windows Movie Maker, Erfindungen und Erfinder,

\section{EINLEITUNG}

Fremdsprachen sind ein wichtiges Medium bei der Kommunikation. Deutsch ist eine der wichtigsten Sprachen in Europa. Um eine fremde Sprache zu lernen, müssen die Studenten vier Kompetenzen beherrschen, diese sind; Sprechfertikeit, Hörverstehen, Leseverstehen und Schreibfertigkeit. Deutsch ist eine Fremdsprache, die an der staatlichen Universität von Medan gelernt wird. Die Lehrbücher sind Studio d A1, A2 und B1. 
Im Buch Studio d A2 gibt es einige Themen, diese sind Sprachen und Biografien, Familienalbum, Reisen und Mobilität, Aktiv in der Freizeit, Medien, Ausgehen, Zu Hause, Kultur erleben, Arbeitswelten, Feste und Geschenke, Mit allen Sinnen sowie Erfindungen und Erfinder. In dieser Studie wird das Thema „Erfindungen und Erfinder” diskutiert. Die Studenten lernen das Thema „Erfindungen und Erfinder” im dritten Semester.

Das Thema Erfindungen und Erfinder ist ein sehr interessantes Thema im Buch Studio d A2, weil es die Entdeckungen aus Deutschland bespricht, zum Beispiel das Aspirin, das von Felix Hoffmann entdeckt wurde, und Das MP3Format, das vom Fraunhofer Institut entwickelt wurde. Mit diesem Thema kann die Motivation der Studenten beim Lernen der deutschen Sprache erhöht werden. Lernmedien können die Lernmotivation verbessern, zum Beispiel Windows Movie Maker, Power Point, Auto Play und Macromedia flash..

Nach der Meinung von Gerlach und Ely (in Arsyad, 2013:3) ist das Medium die Materie, die die Stimmung der Studenten beim Unterricht anhebt. Das kann die Kenntnis und die Fähigkeit der Studenten erhöhen, darüber hinaus kann das Medium auch die Gedanken, Gefühle und die Aufmerksamkeit der Studenten anregen, um sie zum Lernen zu motivieren. Ohne Medium ist der Prozess des Lernens nicht optimal.

Zurzeit gibt es Möglichkeiten, die beim Lernen verwendet werden, um einen interessanten Unterricht $\mathrm{zu}$ gestalten. Eine davon ist der Einsatz von Technologie. Die Entwicklung der Informations und Komunikationstechnologien hat viele verschiedene Produkte der Technologien hervorgebracht, die den Studenten beim Lernen helfen können. Eine davon ist der Windows Movie Maker. Windows Movie Maker ist eine Bearbeitungssoftware, die mehrere Elemente wie Zeichnen, Musik, Schreiben, Animation und Video kombiniert, die interessant für Lernmedien sind. Der Windows Movie Maker ist eine Video-editing-Software, die in der Regel auf jedem Computer installiert ist. Birney (2000) sagt, dass es eine 
Software als Teil des Windows Live Essentials 2011 ist. Der Windows Movie Maker macht mehr Spaß als Lern-Tool im Vergleich zu einem Lehrbuch.

Daher hat sich die Verfasserin dazu entschlossen. die Untersuchung mit dem Titel ist "Die Erstellung des Lermediums zum Thema „Erfindungen Und Erfinder,, aus dem Buch Studio d A2 mit Windows Movie Maker durchzuführen.

\section{THEORETISCHE GRUNDLAGE}

\section{Das Erstellungsmodell}

Richey und Klein (in Sugiyono, 2016:28-29) erklären, dass "Design and development Research is the systematic study of design, development and evaluation processes with the aim of establishing an empirical basis for the creation of instructional and noninstructional product and tool and a new or enhanced model that governs their development". Nach der Meinung kann es geschlossen werden, dass eine Design und Entwicklungsuntersuchung eine systematische Untersuchung ist, wie man das Design eines Produkts entwickelt, und die Leistungsfähigkeit der Produkte bewertet, um die empirischen Daten zu erhalten, und die Mittel und Modelle zum Unterricht zu erschaffen.

In dieser Studie wird die Theorie von Richey und Klein angewendet, um Lernmaterial mit dem Windows Movie Maker zu entwickeln.

In diesem Fall erklären Richey und Klein (in Sugiyono 2016:39) „The focus of Design and Development Research can be front-end analysis. Planning, Production, and Evaluation (PPE)", das heisst, der Schwerpunkt der Entwicklungsuntersuchung ist die Analyse der Design vom Anfang bis zum Ende, die Design, Produktion und Auswertung umfasst.

Die Schritte der Theorie von Richey und Klein sind:

\section{a. Planung}

Planung ist eine Tätigkeit, die Erstellung eines Produkt-Plans für einen bestimmten Zweck zu entwerfen. Die Planung beginnt mit einer Bedarfsanalys und wird durch Forschung und Studium der Literatur durchgeführt. 


\section{b. Die Herstellung}

Die Herstellung ist eine Tätigkeit, das Produkt nach dem Entwurf zu realisieren.

c. Evaluation

Die Evaluation ist ein Test, um zu beurteilen, wie hoch die Qualität das Produkts ist oder ob die Kriterien eines guten Lernmediums schon erfüllt sind.

\section{Die Lernmedien}

Suranto (in Sutirman 2013:15) meint, dass die Medien ein Mittel sind, mit dem die Nachricht eines Komunikators an die Kommunikanten vermittelt wird. Heinich und Freunde (in Sutirman 2013:15) interpretieren die Medien als Vermittler, die Informationen von der Quelle zum Empfänger übertiagen. Also das Fernsehen, Filme, Fotos, Radio, Audioaufnahmen, und Drucksachen gehören zu den Medien.

Gerlach und Ely (in Arsyad 2013:3) behaupten, dass im Allgemeinen Medien als, Material oder Ereignisse verstanden werden, die Bedingungen $\mathrm{zu}$ bauen, die Studierenden Kenntnisse, Fähigkeiten oder Einstellungen zu machen. Sutirman (2013:15) stellt fest, dass Lernmedien als Werkzeuge der Grafik, elektronische, oder Photografis sind, die verwendet werden können, um zu erfassen, $\mathrm{zu}$ verarbeiten und visuellen und verbalen Informationen zu ordnen.

Basierend auf der definition von einigen Experten kann zusammengefasst werden dass ein Lernmedium etwas ist, das eine Nachricht liefern kann, und es kann den Geist, Gefühle und die Bereitschaft der Studenten anregen, um die Schaffung des Lernprozesses im Selbstlernern zu fördern.

\section{Das Buch Studio d A2}

Das Buch Studio d A2 ist ein Buch um Deutsch als Fremdsprache zu lernen. Das Kursbuch gliedert sich in zwölf Einheiten mit thematischer und grammatischer 
Steigerung, die jeweils durch einen Übungsteil und eine Überblickseite ergänzt werden.

\section{Das Thema „Erfindungen und Erfinder”}

Erfindungen und Erfinder ist ein der Themen in dem Buch Studio d A2. Dieses Thema wird im 3. Semester gelernt. In dieser Einheit lernen die Studenten, Beschreibung von Produkten und Erfindungen zu verstehen, welche Dinge man oft benutzt (und wozu), mit Sachtexten arbeiten, und Grammatik (Nebensätze: um zu/ damit, und Passiv mit werden/wurden).

\section{Der Windows Movie Maker}

Der Windows Movie Maker ist eine Video-editing-Software, die in der Regel auf jedem Computer installiert ist. Dieses Program mist im WindowsBetriebssystem integriert. Der Windows Movie Maker ist eine Software, um Audio und Video von der Webkamera, Videokamera oder eine anderen Videoquelle zu erfassen und zu bearbeiten. Darüber hinaus kann man Audio, Video, Fotos oder Bilder mit dem Windows Movie Maker importieren um sie im Film zu verwenden.

\section{Die Schritte der Erstellung des Lernmediums mit den Windows Movie}

\section{Maker}

Windows Movie Maker ist Windows Movie Maker Version 16.4, die im Jahr 2012 veröffentlicht wurde. Dieser Windows Movie Maker ist die Zugabe von Funktionen wie die direkte Aufnahme einer Webcam, HD-Video-Unterstützung, und veröffentlichen auf Facebook, YouTube, MSN, Vimeo, Grub. Windows Movie Maker ist sehr praktisch und jünger bei der Verwendung der anderen Movie Maker.

Das Nutzungprozess der Windows Movie Maker ist:

1. Zuerst öffnet man den Windows Movie Maker.

2. Danach klickt man auf ,judul", um eine Titelseite und Information über die Verfasserin schreiben. 
3. Danach klickt man auf "Tambah video dan foto", um die Fotos in das Programm zu laden. Nachdem das Bild zu wählen, dann klickt man auf "Open".

4. Dann erscheint die Anzeige wie unten.

5. Funktionen für die Bildbearbeitung. (1) Funktion zum Aufnehmen und Hinzufügen von Videos über die Kamera, Ändern von Musik oder Sound und Text (fügen Sie Text aus jedem Bild). (2) die Funktion der Bilder $\mathrm{zu}$ ändern, wie wollte machen filmische Bilder, temporäre Effekte, Pan und zoom-Maus in diese Richtung zu sehen, wie dieses Feature sichtbar ist.

6. Um den Text zu schreiben, klickt man auf "Keterangan" und tippen Sie den Text in "Masukkan teks disni" ein.

7. Um die Textart zu wählen, klicken Sie „Setelan font” und klicken Sie symbol „V” wie in der folgenden Abbildung. Es gibt viele Textarten, man kann einer von denTextarten klicken.

8. Um die Textfarbe zu wählen, wird das Symbol der Farbe geklickt. Wie in der folgenden Abbildung. Dann klickt man eine der Farben und "Efek".

9. Danach klickt man "animasi" für die Videobearbeitung. Sehen Sie in Abbildung 1 ist die dünnen schwarzen Linien, die Teile des Bildes zu organisieren, die Sie Efect hinzufügen möchten.

10. Dann klickt man „Rekaman narasi”, um Schall aufzunehmen und hinzuzufügen. Drücken Sie dann auf "Open".

11. Man klickt auf "Edit ", um die Dauer des Bildes anzupassen. Danach klickt Man auf "Durasi", um die Dauer des Bildes zu bestimmen.

12. Man klickt auf "Tambah Musik”, zum Abrufen von Dateien, die als Hintergrundmusik im Video verwendet werden. Wenn das Lied schon ausgewählt wurde, klickt man auf ,,open “.

13. Dann erscheint die Anzeige wie unten.

14. Wenn man fertig ist, kann man das Video mit AVI-format speichern. Man klickt „Simpan film“ und dann klickt man „Untuk komputer“. 
Danach schreibt man Videotitel. Dann klickt man „save" um das Video zu speichern.

15. Dann erscheint die Anzeige wie unten.

\section{KONZEPTUELLEN GRUNDLAGE}

Basierend auf der zuvor erläuterten theoretischen Grundlage, bieten die Konzeptuellen Grundlagen die passenden Konzepte zum dargestellten Problem. Die Verwendung eines interessanten Lermediums ist sehr wichtig zu beobachten, weil gute Lernmedien die Motivation und Aufmersamkeit der Deutschlernendn beim Lernprozess erhöhen können. Der Windows Movie Maker ist eine Software, um Audio und Video von der Webkamera, Videokamera oder andere Videoquelle zu erfassen, dann die Ergebnisse der Erfassung wird in einen Film bearbeiten. Die Entwicklungsmodell von Richey und Klein besteht aus drei Schritten, nämlich Planung, Herstellung und Evaluation. Mit Windows Movie Maker kann die Motivation der Studenten beim Lernen der deutschen Sprache erhöht werden. Es kann also geschlussfolgert werden, dass gute Lernmedien die Motivation und Aufmersamkeit der Deutschlernendn beim Lernprozess erhöhen können. Die Theorie der Entwicklung von Lernmaterial von Richey und Klein kann Forschern bei der Erstellung mit Windows Movie Maker helfen.

\section{UNTERSUCHUNGSMETODE}

Diese Untersuchung ist eine Entwicklungsuntersuchung. In dieser Untersuchung wird mit dem Windows Movie Maker ein Lernmedium mit dem Thema „Erfindungen und Erfinder” erstellt. Diese Untersuchung benutzt die Theorie von Richey und Klein. Diese Untersuchung benutzt die deskriptivqualitative Methode.

\section{Die Daten und Die Datenquelle}

Die Daten in dieser Untersuchung sind Bilder und Wörter mit dem Thema Erfindungen und Erfinder aus dem Buch Studio d A2. In Kapitel 12 auf Seite 192 bis 205 enthalten. Diese Untersuchung wird in der Bibliothek der Fakultät für Sprache und Kunst an der staatlichen Universität Medan durchgeführt. 
Diese Untersuchung benutzt die Theorie von Richey und Klein. Diese Theorie wurde in Kapitel II erklärt. Diese Theorie besteht aus drei Schritten

\section{ERGEBNIS DER UNTERSUCHUNG \\ Der Prozess der Erstellung}

\section{Die Planung}

Dies ist die erste Phase, um ein Lernmediums zum Thema „Erfindungen und Erfinder" zu erstellen. In dieser Phase wird eine Beobachtungüber die Verwendung des Lernmediums im Unterricht gemacht. Bei dieser Untersuchung erstellt die Verfasserin das Lernmedium mit Windows Movie Maker, das für den effektiven und interessanten Unterricht verwendet wird. Durch ein Windows Movie Maker können die Studenten ohne Büchern efektiv lernen.

Das Thema „Erfindungen und Erfinder” wird von Studenten im dritten Semester gelernt. Die Daten werden von Studio d A2 und Internet genommen. Nachdem alle Materialien gesammelt wurden, werden sie geordnet und zusammengefasst. Das Thema „Erfindungen und Erfinder” handelt von: Aspirin, Der Dieselmotor, Die Strassenbahn, Der Kaffeefilter, Der Buchdruck, Der Teebeutel, Die Zahnpasta, Das Fernsehen, Das MP3-Format, Der Klettverschluss, Die Schiffsschraube, Das Motorrad, Das Automobil, Der Hubschrauber, Der Dübel, und Grammatik ( Nebensätze um zu/damit, Konjugation Verb “warden”, Passiv mit warden/wurden und Übungen.

\section{Das Design}

In der Designphase wird die Materie versammelt. Die Materie besteht aus die Bildern, die Texten und die Übungen mit dem Thema ''Erfindungen und Erfinder', die aus dem Buch Studio d A2, Internet und einigen Website genommen werden. Im Videos gibt es einige Übungen, sie sind: passende Bilder zum Texte einstellen, Textergänzung und Textkaraoke. In dieser Phase wird das Konzept des Lernmediums mit Windows Movie Maker entworfen, um ein interessantes Lernmedium zu erstellen. 
In dieser Phase wird das Konzept des Lernmediums mit dem Video entworfen, um ein interessantes Lernmedium zu erstellen. Alle Teilen in der Windows Movie Maker werden entworfen. Das Design wird an das Thema „Erfindungen und Erfinder” angepasst, das in dem Video integriert wird.

Das Konzept des Designs für diese Windows Movie Maker ist:

$$
\begin{array}{ll}
\text { Das erste Bild } & =\text { Das Aspirin }(\text { Text, Bild und Ton) } \\
\text { Das zweite Bild } & =\text { Das Automobil (Text, Bild und Ton) } \\
\text { Das dritte Bild } & =\text { Der Buchdruck (Text, Bild und Ton) } \\
\text { Das vierte Bild } & =\text { Der Klettverschluss (Text, Bild und Ton) } \\
\text { Das fünfte Bild } & =\text { Der Dieselmotor (Text, Bild und Ton) } \\
\text { Das sechste Bild } & =\text { Der Düdel (Text, Bild und Ton) } \\
\text { Das siebte Bild } & =\text { Das Fernsehen (Text, Bild und Ton) } \\
\text { Das achte Bild } & =\text { Der Hubschrauber (Text, Bild und Ton) } \\
\text { Das neunte Bild } & =\text { Kaffeefilter (Text, Bild und Ton) } \\
\text { Das zahnte Bild } & =\text { Das Motorad (Text, Bild und Ton) } \\
\text { Das elfte Bild } & =\text { Das MP3-Format (Text, Bild und Ton) } \\
\text { Das zwölfte Bild } & =\text { Die Schriffsschraube (Text, Bild und Ton) } \\
\text { Das dreizehnte Bild } & =\text { Die Strassenbahn (Text, Bild und Ton) } \\
\text { Das vierzehnte Bild } & =\text { Der Teebeute (Text, Bild und Ton) } \\
\text { Das fünfzehnte Bild } & =\text { Die Zahnpasta (Text, Bild und Ton) }
\end{array}
$$

\section{Die Herstellung}

Basierend auf der ersten phase wird das Lernmedium erstellt. der Zweite Schritt in dieser Untersuchung ist die Produktion.

\section{a. Die Startseite der Flip Book Maker Software}

Um das Programm der Windows Movie Maker zu bedienen will am Anfang die Startseite Windows Movie Maker geöffnen werden. Dann wird die Taste 
Windows Movie Maker gedrückt. Nach dem drücken wird die Tafel Windows Movie Maker getaucht, wie in der folgenden Abbildung.

\section{b. Die Schritte der Erstellung mithilfe Windows Movie Maker}

In dieser Phase wird eines Lernmedium mithilfe Windows Movie Maker erstellt. Unten sind die Schritte der Erstellung Eines Lernmedium.

1. Windows Movie Maker wird geöffnet. Danach wird auf ,judul'geklickt, um eine Titelseite und Information über die Verfasserin geschrieben.

2. Um Bilder und Fotos zu erfassen, wird "Tambah video dan foto" gewählt. Dann erscheint die Anzeige wie unten.

3. Um den Text zu schreiben, wird auf "Keterangan"geklickt. Danach wird auf „Setelan font" geklickt. wie in der folgenden Abbildung.

4. Um die Textfarbe zu wählen, wird das Symbol der Farbe geklickt. Dann wird die rot Farbe geklickt.

5. Danach wird auf Diagonal-kotak keluar im "animasi" geklickt, für die Videobearbeitung. Wenn animasi schon ausgewählt wurde, dann erscheint die Anzeige wie unten.

6. Dann wird auf „Rekaman narasi” geklickt, um Schall aufzunehmen und hinzuzufügen. Dann wird auf "Open" geklickt.

7. Danach wird auf "Edit" geklicht, um die Dauer des Bildes und Ton anzupassen. Danach wird auf "Durasi" geklicht, um die Dauer des Bildes und Ton zu bestimmen.

8. Dann wird auf "Tambah Musik" geklickt, zum Abrufen von Dateien, die als Hintergrundmusik im Video verwendet werden. Danach wird "Meraih mimpi instrumental” gewählt. Danach wird auf „open“ geklickt.

9. Wenn das Lied schon ausgewählt wurde, dann erscheint die Anzeige wie unten.

10. Nachdem das Video fertig sind, es wird auf „Simpan film“ geklickt. Dann wird auf „Untuk komputer" geklickt. Danach wird auf Datainame geschrieben. Dann wird auf ,save" geklickt, um das Video zu speichern. 
11. Dann erscheint die Anzeige wie unten.

\section{Die Evaluation/Bewertung und Revision}

Dies ist der letzte Schritt, um ein interessantes Lernmedium zu erstellen. In dieser Phase werden die Prüfung und die Validierung durchgeführt, damit herausgefunden werden kann, ob das Lernmedium schon gut genug ist oder noch verbessert werden sollte. Dieses Lernmedium wird von Experten geprüft. Die Experten sind Dozentin und andere Experten, die sich mit dem Design auskennen. Die Validierung des Lernmediums besteht aus zwei Teilen. Zuerst wird das Design evaluiert, das von Experten des Designs gemacht wird.

Der Experte hat auch das Lernmedium mit Windows Movie Maker benotet: Von 10 Kategorien der Aspekte der Evaluation gibt der Experte die Note 4 für die sechs Aspekte, sie sind: Übereinstimmung mit dem Thema, Tonqualität in Windows Movie Maker, das Schreiben der Wörter der deutschen Sprache, Produktdesign, die Klarheit des Materials und die Leichtigkeit des Verständnisses. Die Note 4 bedeutet sehr gut. Der Experte gibt die Note 3 für Bildqualität in Windows Movie Maker, Eignung der Farben im Bild, Videoqualität, die Vollständigkeit der Materialien. Die Note 3 bedeutet gut. Nach der Validierung wird das Lernmedium verbessert.

Basierend auf der obigen Meinung kann festgehalten werden, dass das Lernmedium mit Windows Movie Maker interessant gestaltet ist und besonders gut für das Thema „Erfindungen und Erfinder” ist. Danach können die Videos publiziert werden.

\section{Das Ergebnis der Erstellung}

Das Design des Lernmediums ist vielfältig und kann auf unterschiedliche Weise entworfen werden. In diesem Video gibt es Erklärungen von 15 Entdeckungen aus Deutschland: das Aspirin, das Automobil, der Buchdruck, der Klettverschluss, der Dieselmotor, der Dübel, das Fernsehen, der Hubschrauber, der Kaffeefilter, das MP3-Format, das MP3-Format, die Schiffsschraube, die Strassenbahn, der Teebeutel, die Zahnpasta. Im Video gibt es auch Grammatik 
(Nebensätze um zu /damit, Konjugation des Verbs “werden”, Passiv mit werden/wurden und Übungen. Das Video dauert 13.00 Minuten.

\section{Diskussion}

Basierend auf den Ergebnisse des Lernmediums mit Windows Movie Maker wurde zusammengefasst, dass die Daten in dieser Untersuchung Bilder und Wörter aus dem Buch Studio d A2, Internet und einigen Website genommen werden. In dieser Untersuchung wird die Rickey und Klein Theorie benutzt. Diese Theorie besteht aus drei Stufen, nämlich: (1) die Planung, (2) die Herstellung, (3) Evaluation/Bewertung und Revision.

Basierend auf die obigen Erklärung kann zusammengefasst werden, dass die Erstellung des Lernmediums zum Thema ,, Erfindungen und Erfinder” auf dem Buch studio d A2 mit Windows Movie Maker mit der Rickey und Klein sehr gut ist. Sodass die Dozenten oder die Studenten das Lernmedium benutzen konnen.

\section{SCHLUSSFOLGERUNG}

Nach den Untersuchungsergebnissen fallen die Schlussfolgerungen folgendermaßen aus:

1. Der Prozess der Erstellung eines Lernmediums zumThema „Erfindungen und Erfinder" mit Windows Movie Maker besteht aus der Erklärung der Phasen von Richey und Klein. Darunter sind: (1) Die Planung, (2) Die Erstellung, (3) Evaluation/Bewertung und Revision.

2. Das Ergebnis der Erstellung des Lernmediums zumThema „Erfindungen und Erfinder" aus dem Buch Studio d A2 mit Windows Movie Maker sind:

a. In dieser Untersuchung hat ein Lernmedium Windows Movie Maker, das in diese Abschlussarbeit angehängt wird.

b. Das Lernmedium mit Windows Movie Maker über ,Erfindungen und Erfinder" haben von der Experten evaluiert. das Design evaluiert, das von Experten des Designs gemacht wird. Danach wird die Evaluation der Wörter und der Sprache korregiert wird. 
Basierend auf der Bewertung und Meinungen der Experten können geschlossen werden, dass die Videos gut sind. Die Lernmedien werden einer Deutschdozentin als Expertin geprüft. Das bedeutet, dass die Materıe der Lernmaterialien schon korrigiert wurde.

\section{LITERATURVERZEICHNIS}

Arsyad, Azhar. 2013. Media Pembelajaran. Jakarta: PT.Raja Grafindo Persada.

Bill Birney, Seth McEvoy, Matt Lichtenberg. 2000. Microsof Windows Movie Maker Handbook. Microsof Press.

Febrianti Simarmata. 2015. Pengembangan Media Windows Movie Maker dengan Active And Funny Learning Strategy Materi Larutan Penyangga Siswa Kelas Xi Sma Negeri 11 Semarang. Skripsi. FMIPA. Universitas Negeri Semarang: Semarang

Herman Funk, Christina Kuhn, Silke Demme. 2013. Studio d A2: Deutsch als Fremdsprache. Jakarta: Katalis.

Sugiyono. 2016. Metode Penelitian dan Pengembangan. Bandung: Alfabeta.

Sutirman, M.Pd. 2013. Media dan Model-model Pembelajaran Inovatif. Yogyakarta: Graha Ilmu.

http:// Pengertian windows movie maker.wikipedia.org wird am 28 Juli 2017 um 14.30 Uhr gelesen.

http://usm.ptsb.edu.my/index.php/contact-us/tutorial/33-cara-membuat-dan mengedit-video-menggunakan-movie-maker wird am 5 Agustus 2017 um 14.15 Uhr gelesen.

https://deutsch.lingolia.com/de/grammatik/verben/passiv wird am 27 September 2017 um 15.00 Uhr gelesen. 